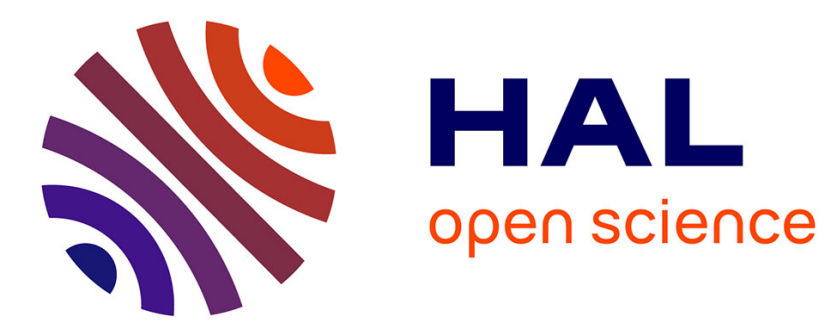

\title{
Spectromètre pour la polarisation dynamique des protons dans un champ de 50 kilogauss
}

\author{
M. Odehnal, P. Néel, V. Bouffard, Cl. Pasquette
}

\section{To cite this version:}

M. Odehnal, P. Néel, V. Bouffard, Cl. Pasquette. Spectromètre pour la polarisation dynamique des protons dans un champ de 50 kilogauss. Revue de Physique Appliquée, 1971, 6 (1), pp.59-63. 10.1051/rphysap:019710060105900 . jpa-00243503

\section{HAL Id: jpa-00243503 https://hal.science/jpa-00243503}

Submitted on 1 Jan 1971

HAL is a multi-disciplinary open access archive for the deposit and dissemination of scientific research documents, whether they are published or not. The documents may come from teaching and research institutions in France or abroad, or from public or private research centers.
L'archive ouverte pluridisciplinaire HAL, est destinée au dépôt et à la diffusion de documents scientifiques de niveau recherche, publiés ou non, émanant des établissements d'enseignement et de recherche français ou étrangers, des laboratoires publics ou privés. 


\title{
SPECTROMẼTRE POUR LA POLARISATION DYNAMIQUE DES PROTONS DANS UN CHAMP DE 50 KILOGAUSS
}

\author{
par M. ODEHNAL (*), P. NÉEL, V. BOUFFARD et Cl. PASQUETTE \\ Service de Physique du Solide et de Résonance Magnétique \\ Centre d'Etudes Nucléaires de Saclay \\ BP no 2, 91, Gif-sur-Yvette, France
}

(Reşu le 21 octobre 1970)

\begin{abstract}
Résumé. - Nous donnons la description d'un appareillage pour la polarisation dynamique des protons dans un champ de $50 \mathrm{kG}$. Les fréquences des spectromètres de résonance paramagnétique électronique et nucléaire sont respectivement $\sim 132 \mathrm{GHz}$ et $202 \mathrm{MHz}$. Le champ magnétique de $50 \mathrm{kG}$ est produit par une bobine supraconductrice. La température de l'échantillon est environ $1{ }^{\circ} \mathrm{K}$. Les résultats de la polarisation des protons dans l'éthylène glycol suggèrent la construction d'un appareil similaire pour une cible opérationnelle de protons polarisés qui peut donner les mêmes performances que les cibles polarisées fonctionnant actuellement à $25 \mathrm{kG}$ et $T \sim 0,50{ }^{\circ} \mathrm{K}$.

Abstract. - A description is given of an apparatus for the dynamic polarization of protons in a field of $50 \mathrm{kG}$. The frequencies of the electron and nuclear paramagnetic resonance spectrometers are $\sim 132 \mathrm{GHz}$ and $202 \mathrm{MHz}$ respectively. The magnetic field of $50 \mathrm{kG}$ is created by a superconducting coil. The temperature of the sample is around $1{ }^{\circ} \mathrm{K}$. The results of the polarization of protons in ethyleneglycol suggest the construction of a similar apparatus for an operating polarized proton target which can give the same performances as the polarized targets now working at $25 \mathrm{kG}$ and $\sim 0,5^{\circ} \mathrm{K}$.
\end{abstract}

I. Introduction. - Les récentes expériences sur la polarisation dynamique des protons à des températures très basses $\left(0,3 \circ \mathrm{K} \leqslant T \leqslant 1,1{ }^{\circ} \mathrm{K}\right)$ ont montré que la polarisation maximale atteinte avec certaines substances (éthylène glycol dopé avec le complexe paramagnétique du $\mathrm{Cr}^{\mathbf{v}}$ ou butanol avec un radical libre porphyrexide $[1,2]$ ) augmente encore notablement lorsqu'on diminue la température absolue de l'échantillon. Ce résultat est très important pour la construction des cibles polarisées utilisées en physique des moyennes et hautes énergies. Déjà l'année 1970 a vu la réalisation de cibles polarisées de grands volumes qui fonctionnent à une température $T$ voisine de $0,5^{\circ} \mathrm{K}$ avec des polarisations d'environ $70 \%$ dans des matériaux riches en protons libres comme le butanol ou l'éthylène glycol [3].

Nous avons voulu explorer l'autre possibilité d'accroissement du rapport $H / T$, où $H$ est le champ magnétique. En maintenant la température de l'échantillon à $T \sim 1{ }^{\circ} \mathrm{K}$, nous avons augmenté le champ magnétique jusqu'à la valeur $47,5 \mathrm{kG}$. A notre connaissance c'est le premier travail sur la polarisation dynamique des protons dans ces conditions et c'est pourquoi il nous semble utile de donner quelques

(*) Adresse permanente : Institut des Recherches Nucléaires, Rež, Tchécoslovaquie. détails sur notre appareillage. Les résultats sur la polarisation des protons dans l'éthylène glycol ont été déjà partiellement publiés ailleurs [4].

Le spectromètre de polarisation dynamique comprend :

- Une bobine supraconductrice donnant un champ magnétique $\sim 50 \mathrm{kG}$.

- Un dispositif cryogénique pour maintenir la température de l'échantillon autour de $1^{\circ} \mathrm{K}$.

- Un spectromètre pour la détection ou la saturation de la résonance paramagnétique des électrons (RPE).

- Un spectromètre pour la détection de la résonance des protons (R.M.N.).

Le schéma d'ensemble est classique et a été maintes fois décrit dans la littérature. Nous donnons ici quelques explications sur les différentes parties du dispositif expérimental.

II. Description de l'appareillage. - 1. BoBINE SUPRACONDUCTRICE. - Le champ magnétique statique $H$ est produit par un solénoïde supraconducteur fabriqué par Oxford Instruments Company Ltd. Le champ au centre est de $55 \mathrm{kG}$ pour un courant d'alimentation de 32,7 A. La transition de la bobine de l'état supraconducteur à l'état normal a lieu pour une 
valeur de champ supérieure à $57 \mathrm{kG}$. Le réglage de l'homogénéité à la valeur souhaitée se fait à l'aide de 2 bobinages latéraux dont les courants peuvent être ajustés séparément, trois interrupteurs supraconducteurs permettant d'isoler les uns des autres bobinage principal et bobinages de compensation. Un fluxmètre très sensible [5] permet d'ajuster les courants dans les trois bobines pour obtenir le champ voulu avec une homogénéité de $10^{-5}$ dans un cylindre de $\Phi=10 \mathrm{~mm}$ et de hauteur $10 \mathrm{~mm}$. Dans notre cas le courant dans les bobines latérales devait être toujours plus grand que celui dans la bobine principale et le réglage d'homogénéité diminuait le champ au centre de quelques centaines de gauss. La figure 1 montre une série de

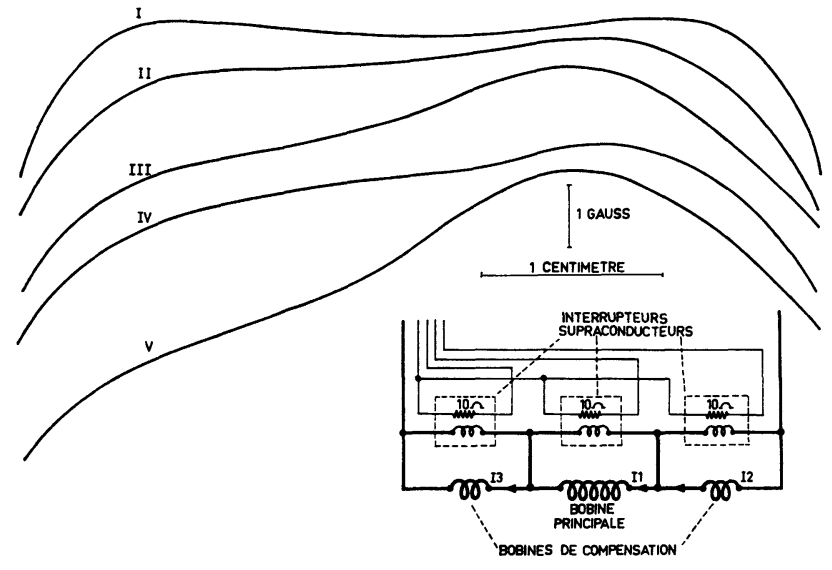

FIG. 1. - Homogénéité de la bobine à 47 kG.

\begin{tabular}{rccc} 
& \multicolumn{3}{c}{ Ampères } \\
& I1 & I 2 & I 3 \\
I & 28,10 & 31,40 & 31,73 \\
II & 28,10 & 31,43 & 31,73 \\
III & 28,10 & 31,60 & 31,73 \\
IV & 28,10 & 31,68 & 31,73 \\
V & 28,10 & 31,73 & 31,73
\end{tabular}

courbes d'homogénéité le long de l'axe de la bobine pour différentes valeurs du courant dans les trois enroulements. Un détail intéressant à noter : la présence d'interrupteurs supraconducteurs en parallèle sur les bobinages provoque une assez importante hystérésis du champ magnétique. Ce phénomène a déjà été observé et la suppression des interrupteurs a diminué nettement cette hystérésis [6]. Pour atténuer son influence et améliorer la reproductibilité du réglage de la valeur du champ, nous avons été amenés à opérer de la façon suivante : avant de se fixer sur la valeur du courant principal $I_{0}$ correspondant au champ voulu, nous effectuons trois cyclages d'amplitude successivement décroissante de part et d'autre de $I_{0}$. $\mathrm{La}$ bobine principale est alors mise en mode persistant et les deux bobines latérales réglées pour obtenir l'homogénéité correcte. Le temps nécessaire à l'ensemble des opérations pour arriver à un champ de $55 \mathrm{kG}$ est de l'ordre de $30 \mathrm{mn}$. En régime persistant la consommation d'hélium liquide du cryostat est de $0,151 / \mathrm{h}$.
La figure 2 montre l'ensemble de la bobine principale (B. P.) avec son cryostat à $4,2^{\circ} \mathrm{K}$ et du cryostat de refroidissement de l'échantillon à $1^{\circ} \mathrm{K}$.

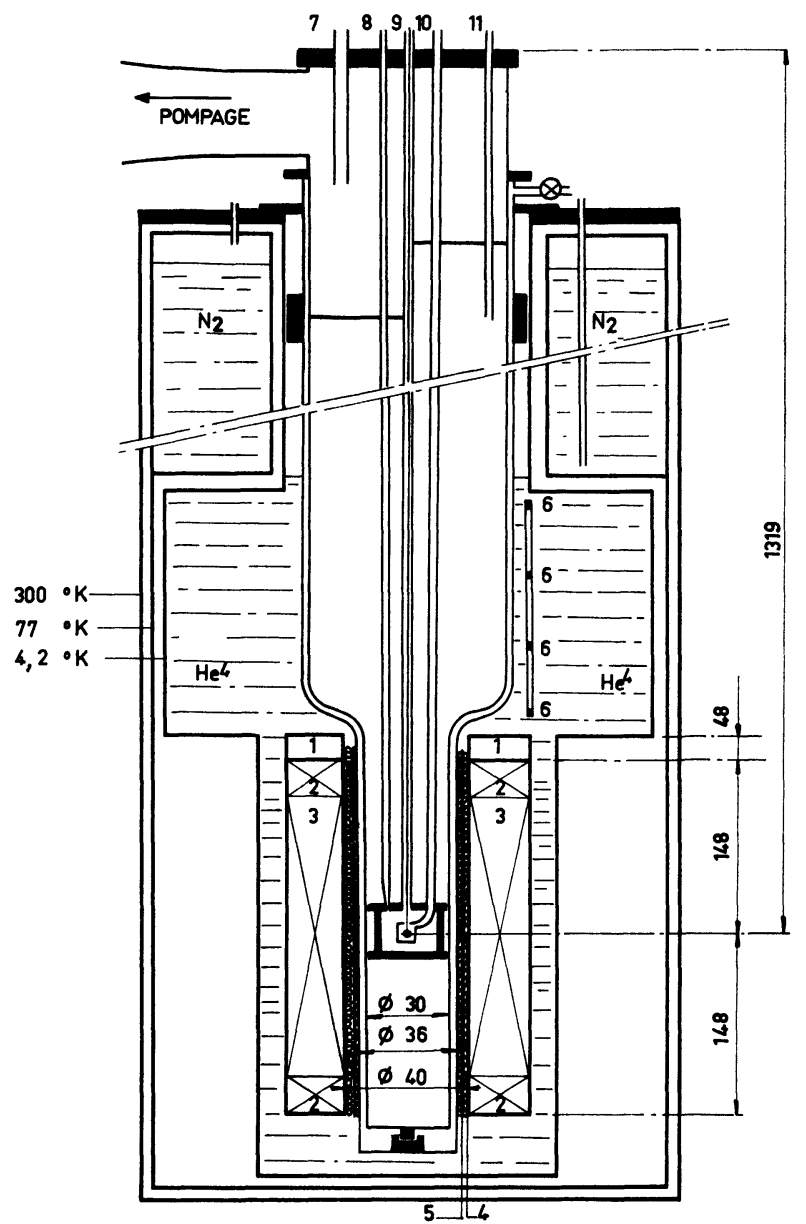

FIG. 2. - Ensemble cryogénique : 1. - Boîte de connexion; 2. - Bobines de compensation; 3.- Bobine principale; 4. - Bobine de balayage; 5. - Bobine de modulation; 6. - Indicateurs de niveau d'hélium ; 7. - Tube de transfert $\mathrm{He}^{4}$; 8. - Guide d'ondes; 9. - Support d'échantillon; 10. - Câble coaxial ; 11. - Mesure de pression.

2. Cryostat a $T=1^{\circ} \mathrm{K}$ et installation de POMPAGE. - Pour travailler à une température voisine de $1{ }^{\circ} \mathrm{K}$, nous avons construit un simple cryostat vertical placé dans le cryostat principal de la $\mathbf{B}$. $\mathbf{P}$. Un remplissage de 4 litres $\mathrm{d}^{\prime} \mathrm{He}^{4}$ assure une autonomie de fonctionnement d'environ 8 heures pour une dissipation de puissance hyperfréquence de quelques dizaines de milliwatts dans la cavité micro-onde. Une canalisation de $\Phi=120 \mathrm{~mm}$ relie le cryostat à une pompe Roots dont la vitesse de pompage est $\sim 1600 \mathrm{~m}^{3} / \mathrm{h}$. Sans puissance hyperfréquence la température de l'échantillon est $T=1,02^{\circ} \mathrm{K}$. Sur la queue de ce cryostat, nous avons bobiné 2 couches de fil de cuivre pour la modulation basse fréquence du champ et quatre couches de fil supraconducteur $\mathrm{Nb}-\mathrm{Ti}$ pour le balayage linéaire du champ principal ( \pm 350 gauss).

3. SPeCtromètre R. P. E. - Ce spectromètre travaille à une fréquence de $130 \mathrm{GHz}(\lambda \sim 2,2 \mathrm{~mm})$. 
Son schéma est sur la figure 3 . Un spectromètre similaire a déjà été utilisé pour des études de polarisation dynamique [7]. Le générateur hyperfréquence est un carcinotron CO-20 de la C. S. F. Ce tube délivre une

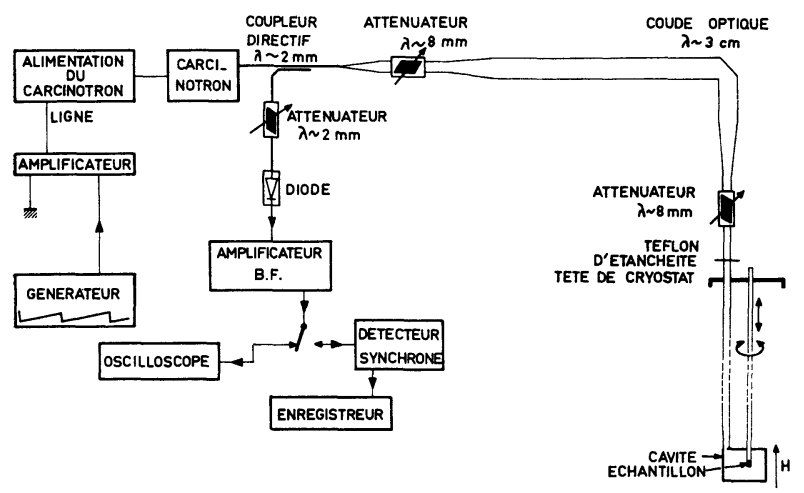

FIG. 3. - Système R. P. E.

puissance maxima de 2,5-3 watts qui dépend de la tension d'anode. La fréquence peut être modifiée en faisant varier manuellement la tension de ligne. La modulation en fréquence est obtenue en injectant entre la masse et la ligne, un signal en dent de scie de fréquence variable entre 50 et $500 \mathrm{~Hz}$ qui produit une modulation en fréquence équivalente à une variation de champ magnétique $\Delta H \sim \pm 5$ gauss. Cette modulation est utile pour les études de polarisation dynamique avec des échantillons de dimension nettement supérieure à la longueur d'onde hyperfréquence, qui doivent être placés dans des cavités multimodes. Dans certains cas cette modulation a entraîné une augmentation notable de la polarisation dynamique.

La transmission de l'onde hyperfréquence se fait dans un ensemble de guides d'onde surdimensionnés de $3 \mathrm{~cm}$ et $8 \mathrm{~mm}$. Pour éviter des pertes supplémentaires $(>3 \mathrm{~dB})$, l'utilisation de coudes optiques est nécessaire. Les portions de guide d'onde $8 \mathrm{~mm}$ en acier inoxydable qui se trouvent dans le cryostat sont intérieurement dorées. Deux atténuateurs $8 \mathrm{~mm}$ peuvent fournir chacun une atténuation supérieure à $10 \mathrm{~dB}$. Les cavités sont cylindriques, de volume $5-10 \mathrm{~cm}^{3}$. Le trou de couplage correspond à la section d'un guide RG 99U $(\lambda \sim 4 \mathrm{~mm})$.

A l'intérieur de la cavité se trouvent la bobine nucléaire et le support de l'échantillon. Dans le cas des expériences avec l'éthylène glycol, une tige vissée au porteéchantillon permettait l'introduction rapide de ce dernier dans le bain $d^{3} \mathrm{He}^{4}$ à $4,2{ }^{\circ} \mathrm{K}$.

La puissance hyperfréquence maxima dissipée par l'ensemble hyperfréquence plongé dans l'hélium liquide était $\sim 350 \mathrm{~m} \mathrm{~W}$ pour une puissance de 2 watts à la sortie du carcinotron. La longueur totale de la ligne hyperfréquence est $\sim 5 \mathrm{~m}$.

Le signal R. P. E. est détecté avec des diodes à $4 \mathrm{~mm}$ ou $2 \mathrm{~mm}$ de la C. S. F. La stabilité en fréquence du carcinotron s'étant avérée suffisante, le circuit de stabilisation en fréquence prévu n'a pas été mis en œuvre.

ReVUe de Physique APPliQuée. - T. 6, No 1, MARS 1971
4. SPeCtromètre R. M. N. - Pour détecter le signal naturel et le signal augmenté des protons, nous avons utilisé un simple Q-mètre de tension. Son schéma de principe est indiqué sur la figure 4. Ajoutons simplement quelques informations complémentaires.

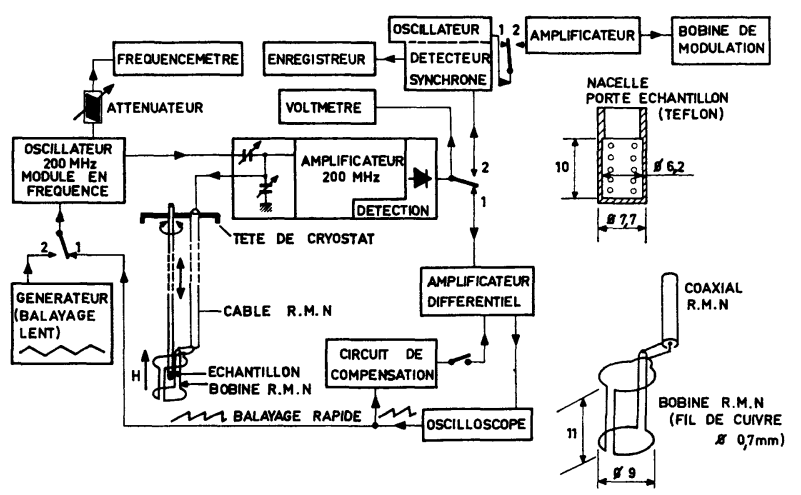

Fig. 4. - Système R. M. N.

La fréquence centrale de l'oscillateur dont le schéma électronique est donné sur la figure 5 , peut être modifiée de $\pm 3 \mathrm{MHz}$ autour de la fréquence moyenne $(\sim 202 \mathrm{MHz})$ en appliquant une tension variable sur une varicap. Un signal en dent de scie appliqué sur la même varicap permet en outre le balayage linéaire de la fréquence de l'oscillateur de $\pm 400 \mathrm{kHz}$. Le niveau de sortie de l'oscillateur est de l'ordre de $0,8 \mathrm{~V}$ sur 50 ohms. La stabilité en fréquence est $\leqslant 10^{-5}$.

Le circuit résonnant est composé d'une bobine nucléaire contenant l'échantillon, d'un condensateur ajustable dans le circuit d'entrée de l'amplificateur et d'un câble coaxial de longueur $\sim \frac{3}{2} \lambda$. La bobine nucléaire dont la forme est détaillée sur la figure 4 est composée de deux spires rectangulaires cintrées sur une surface cylindrique et branchées en parallèle. Une bonne homogénéité du champ radiofréquence est obtenue en choisissant le diamètre de la bobine égal à sa hauteur. Le coefficient de qualité du circuit total est $\sim 50-100$.

La figure 6 montre le schéma de l'amplificateur réalisé avec des triodes céramiques de type 7077 . L'entrée de l'amplificateur est un montage en cascode et les circuits d'accord sont des circuits résonnants série [8]. Le gain HF est $\sim 10^{3}$ et la bande passante est $\pm 2 \mathrm{MHz}$. Une série de condensateurs variables $C_{0}$ accessibles de l'extérieur permet le réglage dans une bande de $\pm 30 \mathrm{MHz}$ autour de la fréquence moyenne.

La détection du signal nucléaire des protons se fait par des procédés classiques, soit par enregistrement avec un détecteur de phase, ce qui nécessite une double intégration pour évaluer la polarisation, soit par photographie du signal sur l'écran de l'oscilloscope en utilisant un balayage rapide et d'amplitude suffisante de la fréquence de l'oscillateur.

Pour des polarisations élevées, afin d'éviter de trop grandes corrections sur le Q-mètre, nous avons toujours choisi des coefficients de remplissage très faibles pour la bobine nucléaire $[9,10]$. Dans le cas des pola- 


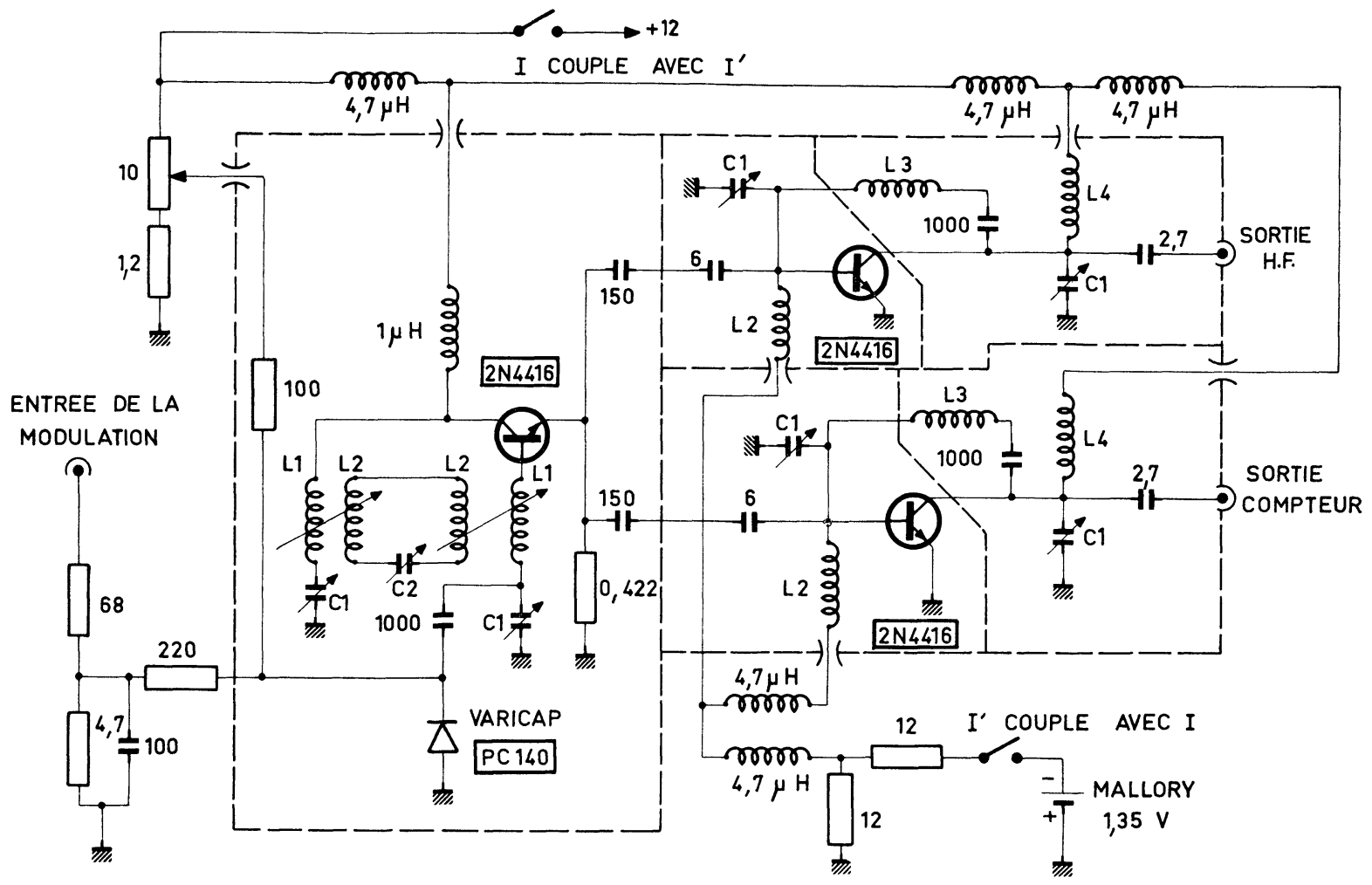

Fig. 5. - Oscillateur 196 à $204 \mathrm{MHz}$; $\breve{C}$ Passage capacitif 1000 pF, R en kohms, C en picofarads. L 1 : 3 Spires fil $\varnothing 1 \mathrm{~s} /$ mandrin $\varnothing 10 ; \mathrm{L} 2: 11 / 2$ Spire fil $\varnothing 1 \mathrm{~s} /$ mandrin $\varnothing 10 ; \mathrm{L} 3: 15$ Spires fil $\varnothing 1 \mathrm{~s} /$ mandrin $\varnothing 10 ; \mathrm{L} 4: 2$ Spires fil $\varnothing 1 \mathrm{~s} /$ mandrin $\varnothing 10 ; \mathrm{C} 1$ : Condensateur ajustable de 0,4 à $12 \mathrm{pF} ; \mathrm{C} 2$ : Condensateur ajustable de 0,4 à $10 \mathrm{pF}$ « Papillon »; Stabilité en fréquence $\leqslant 10^{-5}$. Niveau aux sorties |《 H. F. » et « compteur »: 0,8 volt efficace sur 50 ohms.

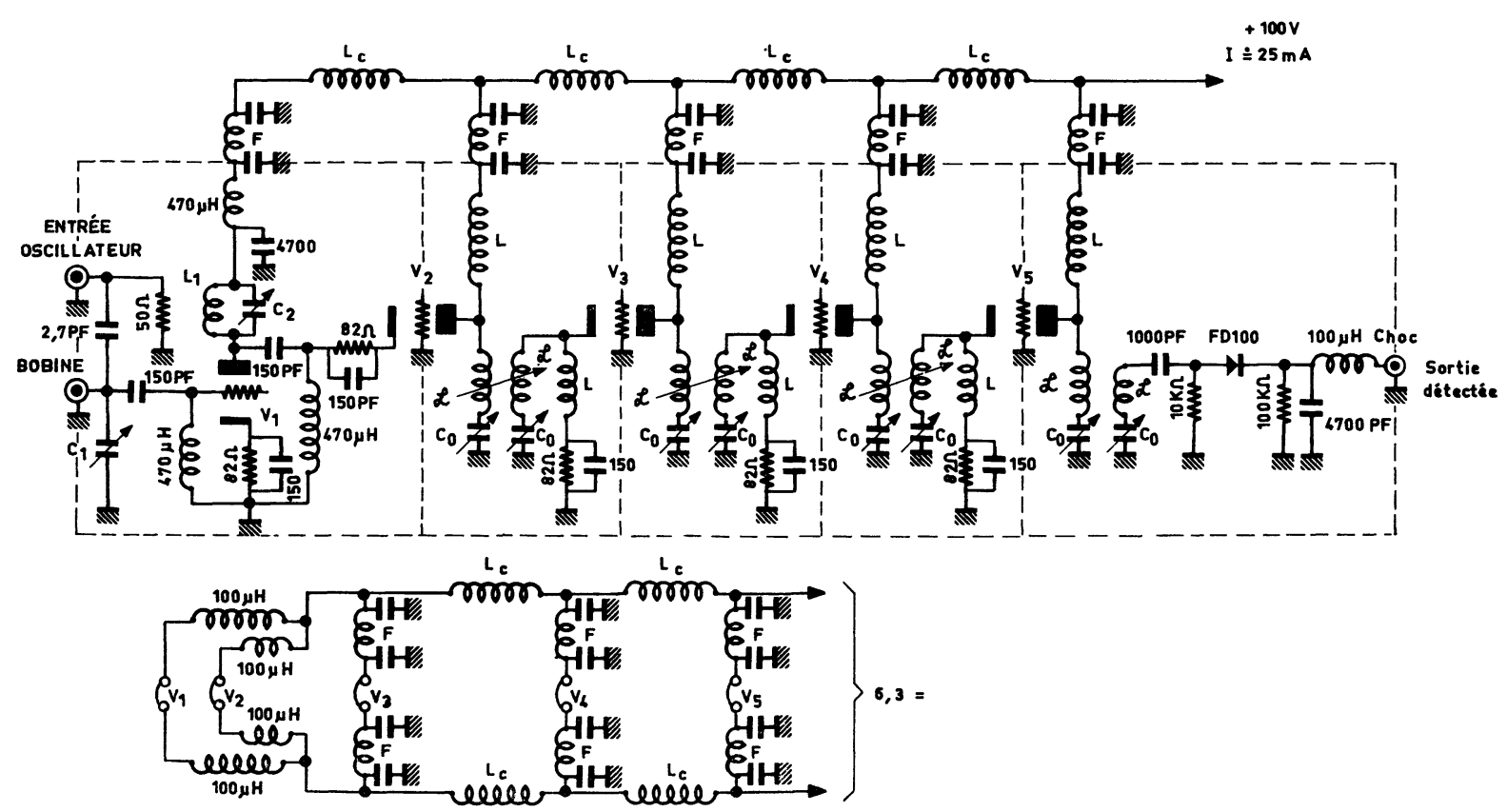

Fig. 6. - Amplificateur Q-mètre $200 \mathrm{MHz}$. Bande passante : $2 \mathrm{MHz}$ à $3 \mathrm{~dB}$; Gain $\mathrm{HF} \sim 1000$. F. Filtres F. C. F. $1122 \times 1000 \mathrm{pF}$ L. C. C. (by Pass); $\mathrm{L}_{\mathrm{c}}$ - - Ferrites d'arrêt HF VK 200 10/3 B Coprim; $C_{1}=C_{2}=\mathrm{COO} 4 \mathrm{EA} / 12 \mathrm{E}$ Coprim $(0,4-12 \mathrm{pF}) ; C_{0}=\mathrm{COO} 4 \mathrm{EA} / 6 \mathrm{E}$ Coprim $(0,4-6 \mathrm{pF}) ;=\mathscr{L} 12$ spires fil émaillé $6 / 10$ bobinées sur $\varnothing=4,1 ; L=$ Choc HF $4,7 \mu \mathrm{H}$ CLO ; $L_{1}=3$ spires $7 / 10$ bobinées sur $\varnothing=4,1$; $V_{1}=V_{2}=V_{3}=V_{4}=V_{5}=7077$. 
risations $\sim 80 \%$, la variation relative de tension à la sortie du détecteur du Q-mètre ne dépassait pas $5 \%$. L'erreur dans l'estimation de la polarisation due à cet effet a été estimée selon la formule :

$$
P_{I}^{ \pm}=P_{I_{0}}\left(1 \pm\left|\frac{\Delta V}{V_{0}}\right|\right) \frac{S}{S_{0}},
$$

où $P_{I}^{ \pm}$est la polarisation nucléaire positive ou négative,

$P_{I_{0}}$ la polarisation d'équilibre thermique sans hyperfréquence,

$S / S_{0}$ le rapport des aires du signal augmenté et du signal naturel,

$\left|\Delta V / V_{0}\right|$ la variation relative de la tension aux bornes du circuit résonnant. De plus la tension radiofréquence a toujours été maintenue à un niveau inférieur à celui de la saturation du signal nucléaire.

5. Conclusions. - Nous avons décrit l'appareillage pour la polarisation dynamique des protons dans des champs magnétiques voisins de $50 \mathrm{kG}$, à la température de $1{ }^{\circ} \mathrm{K}$ et pour des fréquences électroniques et nucléaires voisines de $132 \mathrm{GHz}$ et $202 \mathrm{MHz}$ respectivement. Cet ensemble nous a servi pour des expériences avec des cristaux de $\mathrm{La}_{2} \mathrm{Mg}_{3}\left(\mathrm{NO}_{3}\right)_{12} .24 \mathrm{H}_{2} \mathrm{O}$ dopé au $\mathrm{Nd}^{3+}$ et avec l'éthylène glycol dopé avec le complexe du $\mathrm{Cr}^{\mathrm{V}}$.

Les résultats des mesures ont montré que la polarisation dynamique augmente encore notablement entre $25 \mathrm{kG}$ et $50 \mathrm{kG}$ à $T \sim 1{ }^{\circ} \mathrm{K}$ (surtout dans le cas de l'éthylène glycol où elle passe de $50 \%$ à $80 \%$ ). Ceci permet d'envisager la possibilité de construction d'une cible de protons polarisés avec des performances semblables à celles fonctionnant à $T=0,5{ }^{\circ} \mathrm{K}$ et $H=25 \mathrm{kG}$. Cette cible peut être très avantageuse en physique des hautes énergies pour réaliser des expériences avec des faisceaux intenses de particules chargées, car maintenir la cible à une température voisine de $T=0,5^{\circ} \mathrm{K}$ serait dans ce cas là très difficile à obtenir.

\section{Bibliographie}

[1] Masaike (A.), Glättli (H.), Ezratty (J.) et MaliNowski (A.), Phys. Letters, 1969, 30A, 1.

[2] Hill (D. A.), Ketterson (J. B.), Miller (R. C.), Moretti (A.), NeEMANN (R. C.), Windmiller (L. R.) et Yokosawa (A.), Phys. Rev. Letters, 1969, 23, 9.

[3] Roubeau (P.), Ezratty (J.), Glättli (H.), VerMEULEN (J.) et BORGHINI (M.), Nucl. Instr. and Meth., 1970, 82, 323.

[4] Odehnal (M.) et Bouffard (V.), Phys. Letters, 1970, $32 \mathrm{~A}, 6$.

[5] Grivet (P.), Sauzade (M.) et Stefant (R.), Revue Générale de l'Electricité, 1961, 70, 317.

[6] Schnulin (A. N.), Izvestia A. N. Azerbedjanskoi SSR $n^{\circ} 3,125$ (1969) (en russe).
[7] Odehnal (M.), Loutchikov (V. I.) et Ezratty (J.), J. Physique, 1968, 29, 941.

[8] a) Tube Engineering Bulletin (1959), General Electric (Design considerations and performance factors for the G-E 7077 Ceramic Triode as a low-noise cascode amplifier at 30 and $60 \mathrm{MHz}$ ), Tube Department, Owensboro, Kentucky.

b) Hamilton (M. W.) et RuSh (J. W.), A high performance $500 \mathrm{MHz}$ multi-stage amplifier (1959), General Electric Company, Owensboro, Kentucky.

[9] Borghini (M.), Roubeau (P.) et RYTer (C.), Nucl. Instr. and Meth., 1967, 49, 248.

[10] Petricek (V.) et Odehnal (M.), Nucl. Instr. and Meth., 1967, 52, 197. 